\title{
Uncommon Presentation Of Neisseria Gonorrhoeae In Adults
}

\author{
J Mbuagbaw, E Emilie, N Noa, M Theodosia
}

\section{Citation}

J Mbuagbaw, E Emilie, N Noa, M Theodosia. Uncommon Presentation Of Neisseria Gonorrhoeae In Adults. The Internet Journal of Ophthalmology and Visual Science. 2005 Volume 4 Number 1.

DOI: $10.5580 / \mathrm{c} 8 \mathrm{~d}$

\begin{abstract}
We present cases of acute gonococcocal urethritis/vulvovaginitis associated with involvement of the eye and in some cases the skin and joints observed in four adults in the dermatology clinic of the University Teaching Hospital in Yaounde, Cameroon. Conjunctival and urethral swabs helped us to make the diagnosis. Though rare, we wish to emphasise the importance of considering gonorrhoea as one of the aetiologies and searching for urethritis/vulvovaginitis in patients who present with a copious purulent, conjunctivitis particularly in adults living in an environment where gonorrhoea is still quite prevalent.
\end{abstract}

\section{INTRODUCTION}

Infection due to Neisseria gonorrhoeae, a gram-negative anaerobic diplococcus, is one of the most common sexually transmitted diseases. Although its incidence has dropped markedly, due to better treatment and the use of condoms in the fight against HIV/AIDS, it is still high among homosexual men and commercial sex workers $\left(_{1}\right)$. This latter group constitutes a major source of infections in developing countries.

In adults gonococcal infections are generally localised in the genito-urinary tract. Extra genito-urinary sites involving particularly the eyes are common in children (Gonococcal ophthalmia neonatum) who accidentally may be contaminated by infected mothers at birth $\left(_{2}\right)$.

We report four cases of Gonococcal conjunctivitis seen in adults with concomitant urethritis/vulvovaginitis sometimes associated with articular and skin involvement observed in the dermatology clinic of the University Teaching Hospital in Yaounde.

\section{CASE REPORTS \\ CASE 1}

A 25-year-old male with a history of previous casual sexual contact developed a severe burning sensation during micturition associated with a profuse urethral discharge. Believing his symptoms would clear he sought no medical remedy until two days later when he developed bilateral red discharging eyes with conjunctival haemorrhage, swollen eyelids and photophobia. Pustular lesions with an erythematous base were also found on the limbs on examination. After counselling, urethral and eye swabs as well a blood sample were taken for culture and HIV serologic testing. Culture results revealed Neisseria gonorrhoea from both swabs, sensitive to Ceftriaxone and HIV serology was negative. The eye, urethral discharge and skin eruptions all subsided on treatment with Ceftriaxone. No topical antibiotics were used but daily cleansing of the eyes with normal saline solution was prescribed.

\section{CASE 2}

An otherwise healthy 42-year-old man developed urethral discharge with a burning sensation on micturition. He later noticed a similar bilateral purulent discharge from his eyes and consulted. After clinical examination and counselling, urethral and eye swabs were taken for bacteriologic examination. Blood was also taken for HIV testing. Cultures of the swabs were positive for Neisseria gonorrhoea, sensitive to Ceftriaxone as in Case 1 and recovered completely within one week of treatment.

\section{CASE 3}

A 26-year-old refugee from a neighbouring country was referred to the dermatology clinic of the University Teaching Hospital as a case of Steven Johnson's syndrome induced by 
Cotrimoxazole prescribed for the treatment of dysuria. On examination she still complained of persistent dysuria and had bilateral purulent conjunctivitis, purulent vaginal and urethral discharge as well as features of Steven Johnson's syndrome and arthritis with effusions of the right knee and left ankle. Eye and vaginal swabs were positive for Neisseria gonorrhoea sensitive to Ceftriaxone and HIV serology was positive. She was treated symptomatically with antihistaminics and steroids, for the Cotrimoxazole induced Steven Johnson's syndrome and received a course of Ceftriaxone 1 gram intravenously per day for 7 days for the Gonococcal infection. She recovered completely and was discharged from hospital after 14 days.

\section{CASE 4}

A 32-year-old man presented in the Dermatology clinic with 6 pustular lesions $(5 \mathrm{~mm} \times 4 \mathrm{~mm})$ on an erythematous base. He had an antecedent of casual sexual contact followed by genito-urinary symptoms 8 days prior to consultation. The lesions were located on the limbs and on the chest. He also had monoarthritis of the left knee with effusion, high fever $\left(40.5^{\circ} \mathrm{C}\right)$ and bilateral purulent conjunctivitis. The eye and urethral swabs grew Neisseria gonorrhoea. A gram stain of the material of the pustules was negative but showed degenerated polymorphonuclear cells. Knee effusion was drained; gram stain was negative and with many degenerated polymorphonuclear cells. Culture of the knee tap was not possible. HIV screening was positive. Patient received Ceftriaxone 1 gram IV daily for 7 days and recovered.

\section{DISCUSSION}

Dysuria associated with a purulent urethral discharge commonly seen in males is the commonest presentation of gonococcal infection in our environment. A majority of female patients are generally asymptomatic and are often diagnosed during a contact, tracing or routine investigation for STIs. Gonococcal conjunctivitis is rare in adults and is commonly due to autoinoculation from hand or towels to eye

In adults with confirmed gonococcal conjunctivitis, the infection is usually acquired sexually. Involvement of the conjunctiva is by touching the genital discharge and accidental transfer to the eyes. Patients do not usually relate the purulent eye discharge with their genito-urinary symptoms, and so also the genito-urinary inquiry may be missed out. Gonococcal conjunctivitis is very characteristic. It presents with severe chemosis, corneal oedema, copious purulent exudates and subconjunctival haemorrhage as in
Case 1. If not treated early it can result in corneal ulcer and perforation. In case 3, the involvement of the genitalia and eyes in Steven Johnson's syndrome could result in missing the diagnosis. The diagnosis was suspected because of the copious purulent eye discharge and the persistent dysuria, which antedated the Steven Johnsons syndrome

Other causes of mucopurulent conjunctivitis include Chlamydia trachomatis, Haemophilus species, other pyogenic bacteria, viruses and allergy. These may be excluded when there is no history of recent risky sexual behaviour, no genito-urinary symptoms, and copious purulent conjunctivitis and the non-isolation of intracellular gram-negative diplocci. The two patients each received one gram Ceftriaxone I.M. in a single dose. The eyes were irrigated with normal saline, daily symptoms cleared by the end of the first week.

Disseminated Gonococcal Infection (DGI) occurs in 1 to $3 \%$ of cases and is quite rare. $(3,4)$ DGI results from gonococcal bacteraemia. The lesions are blood born; this dissemination results in pustular skin lesions, which may be predominantly acral, asymmetrical arthralgia, tenosynovitis or septic arthritis. This may be further complicated by perihepatitis, endocarditis or meningitis. These complications of DGI are rare. We had none of these in our patients. It is well known that the strain of Neisseria gonorrhoea that causes DGI may cause minimal genital inflammation.( $\left.{ }_{5}\right)$ The two patients with DGI were infected by the Human Immunodeficiency Virus. Immunodepression may be the principal factor that contributes to the dissemination of the infection.

Other causes of conjunctivitis, urethritis and arthritis should be considered in the differential diagnosis. This would include Reiter's syndrome, septic arthritis and septicaemia. Bacteriology provides the definitive diagnosis. The conjunctivitis is usually transient, mild and may be missed; the arthritis is non suppurative and may involve many more joints as in DGI; while the skin involvement (Keratodermia blemorrheficum) may involve different sites, but most commonly the feet, hands and fingers. Occasionally the lesions may be wide spread and evolve into generalised exfoliative dermatitis. ${ }_{6}$ ) The arthrites may be difficult to differentiate, but gonococcal arthritis does not affect the spine. $\left(_{7}\right.$ ) Bacteriology is important to differentiate these conditions.

All the four patients received oral Doxycycline 100mg twice a day for 14 days for presumptive treatment for Chlamydia infection. Patients infected with Neisseria gonorrhoeae are 
often co-infected with Chlamydia trachomatis $\left(_{8}\right)$. We had no treatment complications.

The main reason for consultations in the four patients are; purulent conjunctivitis in all four, arthritis in 2, skin lesions 2 , Steven Johnson's syndrome 1 and septicaemia 1 . None of the patients had genito-urinary symptoms as a primary complaint. It has been recommended that to avoid missing gonorrhoea infections, cervical swabs, urethral swabs and rectal swabs should be taken for suspicious cases. $\left({ }_{9},{ }_{10}\right)$

These four cases help to stress the role of bacteriology and sensitivity tests in diagnosis of gonococcal infections. All were sensitive to Ceftriaxone. The treatment of uncomplicated gonococcal urethritis and gonococcal urethritis associated with conjunctivitis is intramuscular Cefttriaxone 1 gram in a single dose. $\left(_{11}\right)$ We used this regimen in our patients with good outcome. The patients' eyes were irrigated with normal saline solution four times per day. The two patients with DGI were hospitalised. Each received 1 gram Ceftriaxone intravenously twice a day for 48hours, then 1 gram per day for 7 days. Intramuscularly treatment is also possible. $\left({ }_{10}\right)$ Other recommended drugs include Cefotaxim, Ciprofloxacin, Spectinomycin, Ofloxacin and Cefixime for one week.

\section{CONCLUSION}

Purulent eye discharge in adults should lead to a high suspicion of gonorrhoea in our environment. The need for thorough clinical investigation particularly of the genitourinary system is important. In the rural areas where there are no facilities for culture and sensitivity. One should have a high index of suspicion in any sexually active adult who presents with copious purulent conjunctivitis and dysuria. As it may be difficult to exclude Chlamydia trachomatis infection, treatment should be given for Chlamydia also. In urban areas swabs for bacteriology and sensitivity should be the mainstay for diagnosis of gonococcal infections in our environment. It should also help to differentiate other causes of urethritis, conjunctivitis, arthritis, arthralgia and skin lesions.

\section{References}

1. Hook E.W., Holmes K.K. Gonococal Infections. Ann. Intern. Med. 1985; 102; 229-43.

2. Neinstein L.S., Goldenring J., Carpenter S. Non-sexual transmission of sexually transmitted diseases: an infrequent occurrence. Paediatrics 1984; 74: 67-76.

3. Editorial Disseminated Gonococcal infection. Lancet 1984; 1: 832-3.

4. Holmes K.K., Counts G.W., Beaty H.N. Disseminated Gonococcal infections. Ann Intern. Med. 1971; 74: 979.

5. MMWR May 10 2002/ Vol. 51/ N RR-6 pg 38.

6. Kousa M. Clinical Observations on Reiter's disease with special reference to venereal and non-venereal aetiology. Acta Derm Venereal. 1978; 58 (suppl): 1-36.

7. Hurd F.R., Johns J., Chubick A. Comparative Study of gonococcal arthritis and Reiter's syndrome. Ann Rheum Dis. 1997 ; 38 (suppl) : 55-58

8. MMWR. Recommendations and reports in sexually transmitted diseases treatment. Guideline May 10, 2002/Vol $51 / N^{\circ}$. RR-6.

9. Bhattacharyya M.N., Jephco A.E. Diagnosis of Gonorrhoea in Women. Br. J Vener Dis 1974; 50: 109-12. 10. Schroeter A.L., Reynolds G. The rectal culture as a test of cure of gonorrhoea in the female. Infect Dis 1972; 125 : 499-503.

11. Haimovici R. Roussel T.J. Treatment of gonococcal cojunctivitis with single dose of intramuscular Ceftriaxone. Am J. Ophthalmol 1989; 107: 551-4. 


\section{Author Information}

\section{Josephine Mbuagbaw}

Internal Physician/Dermatologist, Department of Internal Medicine, Faculty of Medicine and Biomedical Sciences, University of Yaounde I

\section{Epee Emilie}

Ophtalmologist, Department of Ophthalmology, University Teaching Hospital University of Yaounde I

\section{Noa Noa}

Ophthalmologist, Department of Ophthalmology, University Teaching Hospital University of Yaounde I

\section{Mcmoli Theodosia}

Ophthalmologist, University of Buea 